\title{
MicroRNA-328 directly targets p21-activated protein kinase 6 inhibiting prostate cancer proliferation and enhancing docetaxel sensitivity
}

\author{
CHUNHUI LIU*, LEI ZHANG* ${ }^{*}$ YEQING HUANG ${ }^{*}$, KAI LU, TAO TAO, SHUQIU CHEN, \\ XIAOWEN ZHANG, HAN GUAN, MING CHEN and BIN XU
}

Department of Urology, Affiliated Zhongda Hospital of Southeast University, Nanjing, Jiangsu 210009, P.R. China

Received February 5, 2015; Accepted September 1, 2015

DOI: $10.3892 / \mathrm{mmr} .2015 .4390$

\begin{abstract}
Prostate cancer (Pca) has one of the highest mortality rates for malignant cancers worldwide. Previous research has demonstrated that numerous genes are aberrantly expressed during Pca onset and development. p21-activated protein kinase 6 (PAK6) is known to be overexpressed in primary and metastatic Pca, however the mechanism of this aberrant expression remains unknown. In the present study, immunohistochemistry demonstrated that PAK6 is overexpressed in castration-resistant Pca (CRPC). Furthermore, PAK6 overexpression was regulated by microRNA (miR)-328 . Luciferase reporter assay and western blot analysis indicated that PAK6 was directly targeted by miR-328. Forced expression of miR-328 enhanced docetaxel sensitivity, inhibited cell proliferation and promoted cell apoptosis without affecting the cell cycle. This indicates that miR-328 performs important functions in CRPC progression via PAK6 regulation. This mechanism may be used to enhance the effect of docetaxel.
\end{abstract}

\section{Introduction}

Prostate cancer (Pca) has one of the highest mortality rates for malignant cancers worldwide. It is the most frequently diagnosed type of cancer and the second leading cause of mortality in males in the USA (1). The majority of Pca-associated mortality is due to metastatic castration-resistant Pca (CRPC). While novel treatments, such as docetaxel, enzalutamide, abiraterone, sipuleucel-T, cabazitaxel and radium-223, have

Correspondence to: Dr Ming Chen or Dr Bin Xu, Department of Urology, Affiliated Zhongda Hospital of Southeast University, 87 Dingjia Bridge Hunan Road, Nanjing, Jiangsu 210009, P.R. China E-mail: mingchenseu@126.com

E-mail: xb15896450810@126.com

*Contributed equally

Key words: castration-resistant prostate cancer, proliferation, p21-activated protein kinase 6, microRNA-328, docetaxel been demonstrated to improve survival for patients with metastatic CRPC, the disease remains incurable (2). To determine more efficacious therapeutic methods, the underlying mechanism of Pca onset, and transition from castration-sensitive Pca to CRPC, must be established.

Molecular changes in Pca have been extensively investigated, and numerous genes have been observed to be aberrantly expressed during Pca onset and development (3). PAK6, a serine threonine kinase that belongs to the PAK family (4), is an androgen receptor-interacting protein (5). PAK6 was recently indicated to be overexpressed in primary and metastatic Pca (6). Furthermore, increased PAK6 expression has been observed in the LAPC4, PC-3 and DU-145 cell lines (7). PAK6 overexpression in tumors may promote cell proliferation and inhibit apoptosis. Previous studies demonstrate that PAK6 knockdown in prostate cell lines inhibits cell growth, and enhances docetaxel chemosensitivity and radiosensitivity $(8,9)$. However, the mechanism of aberrant PAK6 expression remains to be fully elucidated.

Recent research demonstrates that post-transcriptional events perform significant functions in aberrant gene expression. MicroRNAs (miRs) are important molecules involved in post-transcriptional events (10). miRs are small and endogenously produced non-coding RNAs of 19-25 nucleotides in length that negatively regulate target gene expression by binding complementary sequences in the 3'-untranslated region (UTR) of mRNAs, which results in translational repression or direct mRNA cleavage (11). Various miRs have been demonstrated to contribute to Pca by affecting cell development, proliferation, differentiation and apoptosis. miR-143, for example, inhibits Pca cell proliferation and migration, and enhances cell sensitivity toward docetaxel via KRAS downregulation (12).

Considering these previous findings, it is hypothesized that specific miRs participate in PAK6 regulation. Thus, the aim of the present study was to identify an miR that targets PAK6 and determine the function of this miR in Pca.

\section{Materials and methods}

Human Pca specimens. All of the specimens used in the present study were collected from the Affiliated Zhongda Hospital of 
Southeast University (Nanjing, China) between March 2012 and April 2014. Ethical approval was obtained from the relevant ethics committee at the Affiliated Zhongda Hospital of Southeast University. All of the samples were collected upon receipt of written informed consent from patients. Benign prostate hyperplasia (BPH) tissues were obtained from 10 patients who had undergone transurethral prostatic resection (TURP). Pca tissues were obtained from 30 androgen-dependent Pca (ADPC) patients who had undergone radical prostatectomy. All patients were divided into three groups based on a Gleason score of $<7,7$ or $>7$ (13). Nine patients were diagnosed with CRPC, as their serum prostate-specific antigen levels continued to increase despite maximum androgen deprivation therapy. All patients with CRPC were in stage T4 (distant metastasis) and presented with a Gleason score of $>7$ (13). These patients had undergone TURP due to urinary retention. Histological diagnosis was conducted on freshly frozen sections following hematoxylin and eosin staining (Beyotime Institute of Biotechnology, Shanghai, China). Ten ADPC samples with Gleason score $>7$ and $>60 \%$ tumor content and nine patients with CRPC were included in the present study for quantitative reverse transcription-polymerase chain reaction (qRT-PCR). The specimens used for miR qRT-PCR were snap-frozen in liquid nitrogen (Nanjing University Physics Refrigeration Laboratory, Nanjing, China).

Immunohistochemistry. All surgical samples were fixed in 10\% buffered formaldehyde solution (BioSharp, Hefei, China) and embedded in paraffin (Sigma-Aldrich, St. Louis, MO, USA). Paraffin sections ( $4-\mu$ m thick) were reacted with polyclonal antibodies against PAK6 (1:50; cat. no. 13539-1-AP; ProteinTech Group, Inc., Chicago, IL, USA). Phosphate-buffered saline (PBS; Zhongshan Golden Bridge Biotechnology Co., Ltd., Beijing, China) served as a negative control (NC).

$q R T-P C R$. Total RNA was extracted from specimens using TRIzol (Invitrogen Life Technologies, Carlsbad, CA, USA). cDNA synthesis was performed using PrimeScript ${ }^{\circledR} 1$ st Strand cDNA Synthesis kit (Takara Biotechnology Co., Ltd., Dalian, China). qRT-PCR reactions were performed using the SYBR Green PCR Master mix from the Hairpin-it ${ }^{\mathrm{TM}}$ miRs RT-PCR Quantitation kit (GenePharma Co., Ltd., Shanghai, China) according to the manufacturer's instructions. PCR conditions were used to detect miRs as follows: $95^{\circ} \mathrm{C}$ for $3 \mathrm{~min}, 40$ cycles at $95^{\circ} \mathrm{C}$ for $12 \mathrm{sec}$ and $62^{\circ} \mathrm{C}$ for $40 \mathrm{sec}$. The miR-328 expression relative to U6 was calculated using the $2^{-\Delta \Delta \mathrm{Ct}}$ method.

Western blot analysis. Specimens and cells were lysed with radioimmunoprecipitation buffer (Beyotime Institute of Biotechnology). Protein concentrations were determined by the bicinchoninic acid method (Beyotime Institute of Biotechnology). Equal amounts of proteins $(30 \mu \mathrm{g})$ were separated by $10 \%$ SDS-PAGE (Beyotime Institute of Biotechnology) at $80 \mathrm{~V}$ for $30 \mathrm{~min}$ then $100 \mathrm{~V}$ for $1.5 \mathrm{~h}$. Electrophoresed proteins were transferred to a polyvinylidene difluoride membrane (EMD Millipore, Billerica, MA, USA) and subsequently blocked with 5\% skimmed milk (BioSharp) at room temperature for $1 \mathrm{~h}$. The membranes were incubated with rabbit anti-human PAK6 polyclonal antibody (1:400; cat. no 13539-1-AP; ProteinTech Group, Inc.), rabbit anti-human cleaved caspase-3 antibody (1:500; cat. no. 9654; Cell Signaling Technology, Danvers, MA, USA), mouse anti-human caspase-9 monoclonal antibody (1:500; cat. no. 9492; Cell Signaling Technology), rabbit anti-human bcl-2 polyclonal antibody (1:500; cat. no. 12789-1-AP; ProteinTech Group, Inc.), or rabbit anti-human GAPDH polyclonal antibody (1:1,000; cat. no. sc-25778; Santa Cruz Biotechnology Inc., Dallas, TX, USA) in 5\% skimmed milk overnight at $4^{\circ} \mathrm{C}$. The blots were washed with Tris-buffered saline with Tween 20 , incubated with horseradish peroxidase-labeled goat anti-rabbit secondary antibody $(1: 3,000$; cat. no. ZB-2301; Zhongshan Golden Bridge Biotechnology Co., Ltd.) at $37^{\circ} \mathrm{C}$ for $1 \mathrm{~h}$, and visualized using Immobilon Western Chemilum HRP Substrate (EMD Millipore). Protein levels were determined by normalization against GAPDH.

\section{Plasmid construction and luciferase reporter assay. A PAK6} 3'-UTR-luciferase reporter was created by ligating the PAK6 3'-UTR PCR product into the XhoI and NotI restriction sites of the psiCHECK-2 ${ }^{\mathrm{TM}}$ Vector (Promega Corp., Madison, WI, USA). Deletion of the binding site for miR-328 generated the mutant reporter. Following a 48-h cotransfection, luciferase activity was evaluated using a dual-luciferase reporter assay system (Promega Corp.).

Cell culture. The PC-3 and DU-145 human Pca cell lines, and HEK-293 cells were purchased from the Chinese Academy of Sciences Cell Bank (Shanghai, China). HEK-293 and DU-145 cells were cultured in Dulbecco's modified Eagle's medium (DMEM; GE Healthcare Life Sciences, Logan, UT, USA), and PC-3 was cultured in DMEM F12 (GE Healthcare Life Sciences) supplemented with $100 \mathrm{U} / \mathrm{ml}$ penicillin (GE Healthcare Life Sciences), $100 \mathrm{mg} / \mathrm{ml}$ streptomycin (GE Healthcare Life Sciences), and 10\% fetal bovine serum (GE Healthcare Life Sciences). All cell cultures were incubated at $37^{\circ} \mathrm{C}$ in an atmosphere of $5 \% \mathrm{CO}_{2}$.

Oligonucleotides and cell transfection. miR mimic oligonucleotide duplexes were chemically synthesized by GenePharma, Co., Ltd. based on the following sequences: Sense, 5'-CUG GCCCUCUCUGCCCUUCCGU-3' and antisense, 5'-GGA AGGGCAGAGAGGGCCAGUU-3' for hsa-miR-328 mimic; and sense, 5'-UUCUCCGAACGUGUCACGUTT-3' and antisense, 5'-ACGUGACACGUUCGGAGAATT-3' for the NC. For cell transfection, DU-145 and PC-3 cells were seeded in 6-well plates and transfected at $60-70 \%$ confluence using Lipofectamine 2000 (Invitrogen Life Technologies) according to the manufacturer's instructions.

Cell proliferation and cytotoxicity assays. DU-145 and PC-3 cells were seeded in 6 -well plates, cultured at $37^{\circ} \mathrm{C}$ in an atmosphere containing $5 \% \mathrm{CO}_{2}$ overnight, transfected with oligonucleotides, and cultured for a further $48 \mathrm{~h}$. Subsequently, the cells were trypsinized (Gibco; Thermo Fisher Scientific Inc., Waltham, MA, USA) and seeded at a density of 3,000 cells/well (200 ml/well) in 96-well plates. Following overnight incubation at $37^{\circ} \mathrm{C}$ in an atmosphere containing $5 \% \mathrm{CO}_{2}$, the cells were treated with various docetaxel concentrations. Cell proliferation was evaluated 
using a Cell Counting Kit-8 (CCK-8) assay kit (Beyotime Institute of Biotechnology) according to the manufacturer's instructions. Absorbance was detected at $450 \mathrm{~nm}$ using an automatic multi-well MK3 spectrophotometer (Thermo Fisher Scientific, Inc.). Five wells were analyzed for cell viability in each treatment group.

Cell cycle and apoptosis assays. Approximately $48 \mathrm{~h}$ post-transfection, cells were harvested and stained with propidium iodide [PI; MultiSciences (Lianke) Biotech Co., Ltd., Hangzhou, China] for cell cycle assay and by Annexin V-fluorescein isothiocyanate and PI (Ubio Biological Technology PVT Ltd., Jinan, China) for the apoptosis assay, according to the manufacturer's instructions. Treated cells were analyzed by flow cytometry (FACS101; BD Biosciences, Franklin Lakes, NJ, USA).

Tumorigenicity assays in a nude mouse model. Six 4-week-old immunodeficient BALB/c-nu/nu male mice were obtained from Shanghai SLAC Laboratory Animal Co., Ltd. (Shanghai, China) for injection of PC-3 cells. The mice were maintained under specific pathogen-free conditions under a $12 \mathrm{~h}$ light $/ 12 \mathrm{~h}$ dark cycle at $26-28^{\circ} \mathrm{C}$ and $50-65 \%$ humidity. Cell suspensions (100 $\mu 1 ; 5 \times 10^{6}$ cells $)$ were subcutaneously injected into the dorsal scapular region of each mouse. Tumor volume was measured using a caliper every three days and the following formula was used: Volume $\left(\mathrm{mm}^{3}\right)=\left(\right.$ length $\mathrm{x}$ width $\left.{ }^{2}\right) / 2$. When the tumor volume reached $40-50 \mathrm{~mm}^{3}$, the mice were randomly divided into two groups with three mice per group. The mice were treated with 200 pmol NC or hsa-miR-328 mimics in $10 \mu \mathrm{l}$ RNA free water by local injection of the xenograft tumor at multiple sites. This treatment was performed once every five days for 15 days, and tumors were harvested one week later. Animal experiments were undertaken in accordance with National Institute of Health Guide for the Care and Use of Laboratory Animals guidelines and were approved by the ethics committee of the Affiliated Zhongda Hospital of Southeast University.

Statistical analysis. Data from at least three independent experiments are presented as means \pm standard error of the mean. Differences between groups were calculated by Student's t-test or one-way analysis of variance using the SPSS 16.0 software package (SPSS, Inc., Chicago, IL, USA). $\mathrm{P}<0.05$ was considered to indicate a statistically significant difference.

\section{Results}

PAK6 protein expression in tissues. PAK6 expression levels were detected using immunohistochemistry. Cytoplasmic staining was observed, although no nuclear expression was demonstrated in the Pca tissue samples (Fig. 1A). In the BPH tissue samples, PAK6 was predominantly expressed in the epithelium; however, the staining was weak in the majority of BPH tissues (Fig. 1B). In the Pca samples, the staining intensity appears to be greater in groups with Gleason scores of 7 and $>7$, when compared with the group with Gleason score of $<7$ (Fig. 1C-E). The staining intensity was notably increased in the CRPC tissue samples (Fig. 1F). The results of immunostaining are summarized in Table I. PAK6 expression levels were detected by western blot analysis in three pairs of ADPC and CRPC tissues, and overexpression of PAK6 was observed in the CRPC tissue samples (Fig. 1G). These results are consistent with those of a previous study (6).

PAK6 is directly targeted by miR-328. To determine which miR affects PAK6 expression, bioinformatics analysis was performed using TargetScan Human 6.2 (http://www. targetscan.org/) and miRanda (http://www.microrna.org/ microrna/home.do). Of the miRs identified, miR-328 was identified to be bound to the 3'-UTR of PAK6 mRNA with high scores (Fig. 2A). miR-328 expression was subsequently detected in 10 ADPC and nine CRPC tissue samples by qRT-PCR, and weak expression was observed in CRPC tissues (Fig. 2B). In addition, PAK6 protein expression was inhibited in cells transfected with miR-328 mimics (Fig. 2C). These results indicate that PAK6 is the potential target of miR-328. To determine direct miR-target interactions, a luciferase reporter assay was conducted by constructing wild- and mutant-type cells with the luciferase vector. A significant decrease was observed in the luciferase activities of HEK-293 and PC-3 cells when compared with those of the control and mutant-type cells (Fig. 2D). These characteristics indicate that miR-328 directly targets PAK6.

miR-328 inhibits cell growth and promotes cell apoptosis. To investigate the functional roles of miR-328 in Pca progression, miR-328 or NC mimics were transfected into PC-3 and DU-145 CRPC cell lines. NC mimic-incorporated green fluorescent protein demonstrated high transfection efficiency (Fig. 3G). CCK-8 assay and nude mouse transplantation tumor experiments demonstrated that miR-328 overexpression inhibits PC-3 cell proliferation in vivo and in vitro (Fig. 3A-3C). In addition, reduced staining was observed in miR-328-treated xenografts (Fig. 3D). The proapoptotic effect of miR-328 was observed in PC-3 and DU-145 cells by flow cytometry assay (Fig. 3E and F). The same assay, however, indicated that miR-328 does not affect cell cycle progression (data not shown). The viability of caspase-3, -9 and bcl-2 was examined in cells following transfection. Compared with cells transfected with NC mimics, increased cleavage of caspase- 3 and -9 and decreased bcl-2 were observed in cells transfected with miR-328 (Fig. 3H). These results indicate that miR-328 may inhibit cell growth and promote cell apoptosis.

miR-328 enhances docetaxel sensitivity. A previous study demonstrated that PAK6 knockdown in prostate cell lines increases docetaxel sensitivity (8). To determine if miR-328 exerts a similar effect, assessment of docetaxel drug sensitivity following transfection with miR-328 was conducted. Cells were transfected with miR-328 or NC, and treated with docetaxel at concentrations of 5,10, 20, 50 or $100 \mathrm{nM}$ for $24 \mathrm{~h}$. Subsequent to miR-328 transfection, the docetaxel drug concentration that inhibited $50 \%$ of cell proliferation significantly decreased from 25.45 to $8.30 \mathrm{nM}(\mathrm{P}<0.05)$ in $\mathrm{PC}-3$ cells and from 36.63 to $16.78 \mathrm{nM}(\mathrm{P}<0.05)$ in DU-145 cells (Fig. 4). 
A

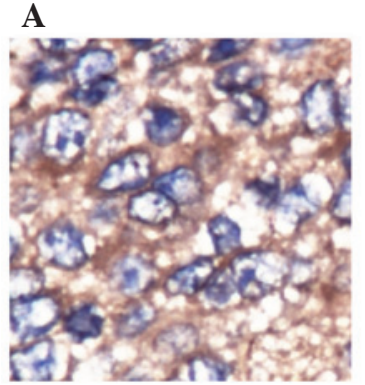

D

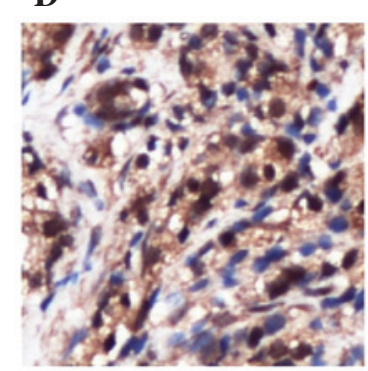

B

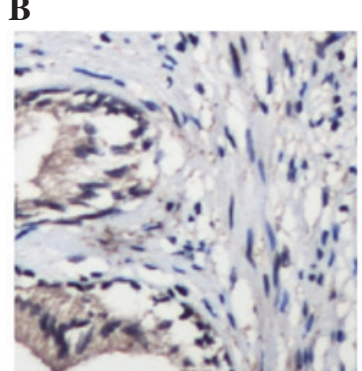

$\mathbf{E}$

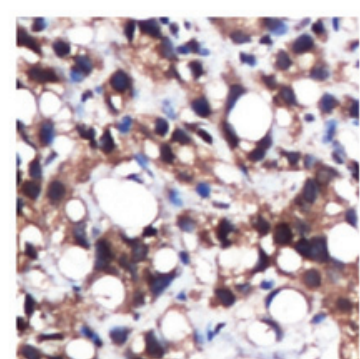

C

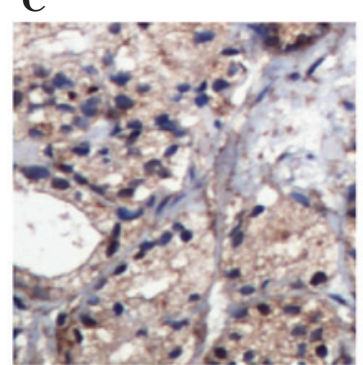

F

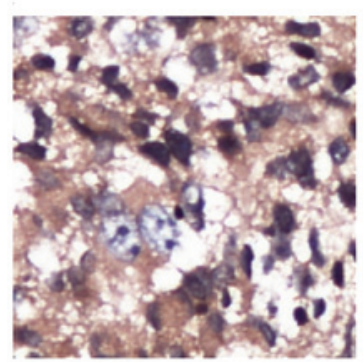

G

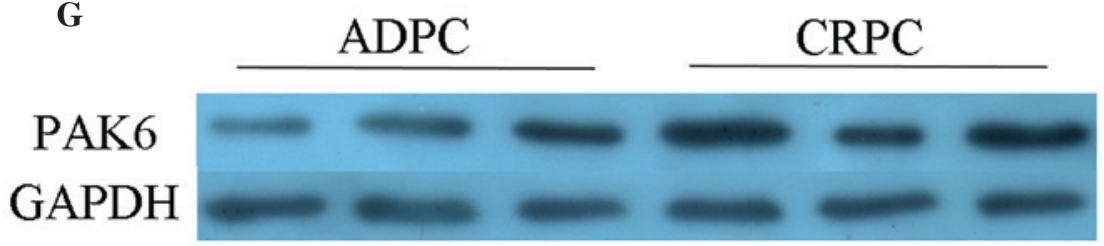

Figure 1. PAK6 expression in BPH and Pca tissue samples. (A) Pca samples were stained with anti-PAK6 (magnification, x400). (B) BPH and (C-F) Pca tissue samples were stained with anti-PAK6 (magnification, x200). Tissue samples with Gleason score of (C) $<7$; (D) 7 and (E) $>7$. (F) CRPC tissues. (G) PAK6 overexpression in CRPC samples was dectected by western blotting. PAK6, p21-activated protein kinase 6; BPH, benign prostate hyperplasia; Pca, prostate cancer; CRPC, castration-resistant Pca; ADPC, androgen-dependent Pca.

A

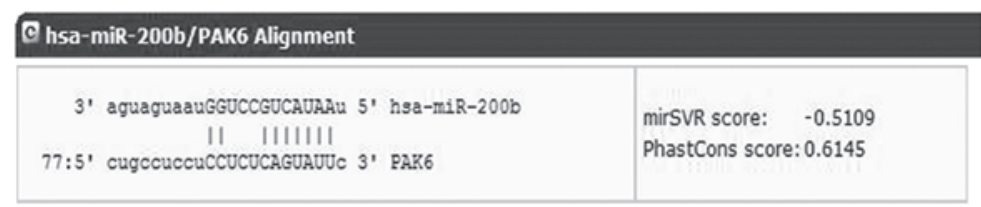

B

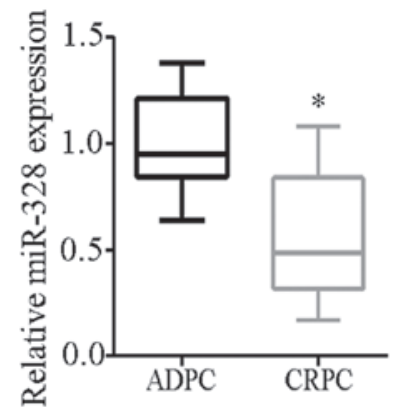

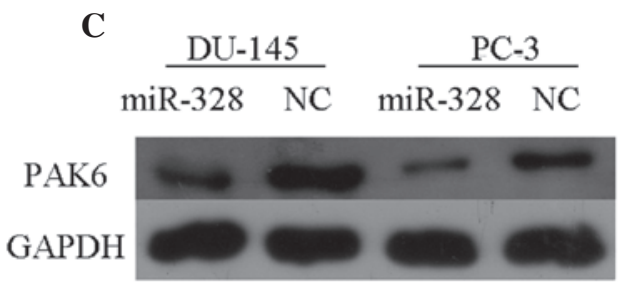

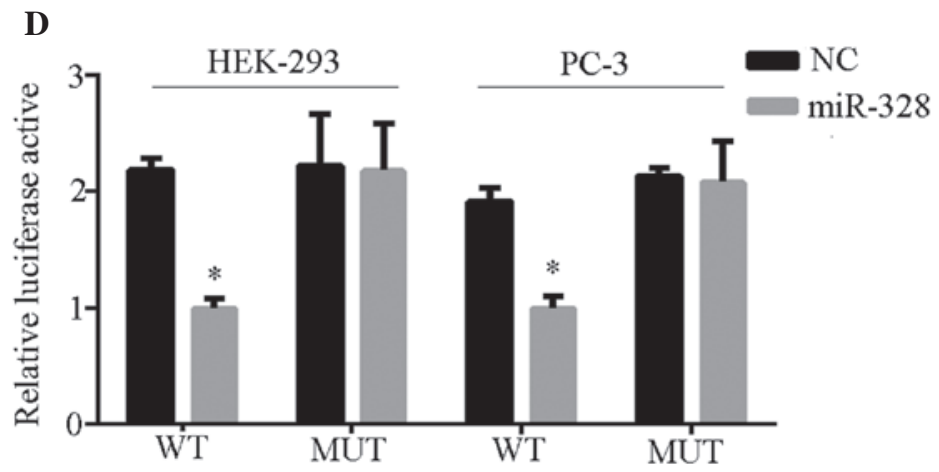

Figure 2. PAK6 was directly targeted by miR-328. (A) miR-328 may bind to target sequences located in the nucleotides of the 3'-UTR of PAK6 mRNA (miRanda). (B) Quantitative reverse transcription-polymerase chain reaction identified decreased miR-328 expression levels in CRPC tissue samples compared with in ADPC tissues ("P<0.05). (C) PAK6 protein was inhibited by treatment with miR-328 mimics, as evaluated by western blotting. (D) PAK6 3'-UTR was cloned into a luciferase reporter cloning site in the psiCHECK-2 dual luciferase vector. Luciferase activity markedly decreased with increasing miR-328 expression level ( $\left.{ }^{*} \mathrm{P}<0.05\right)$. PAK6, p21-activated protein kinase 6; miR, microRNA; CRPC, castration-resistant prostate cancer; ADPC, androgen-dependent prostate cancer; NC, negative control; WT, wild-type; MUT, mutant. 
A
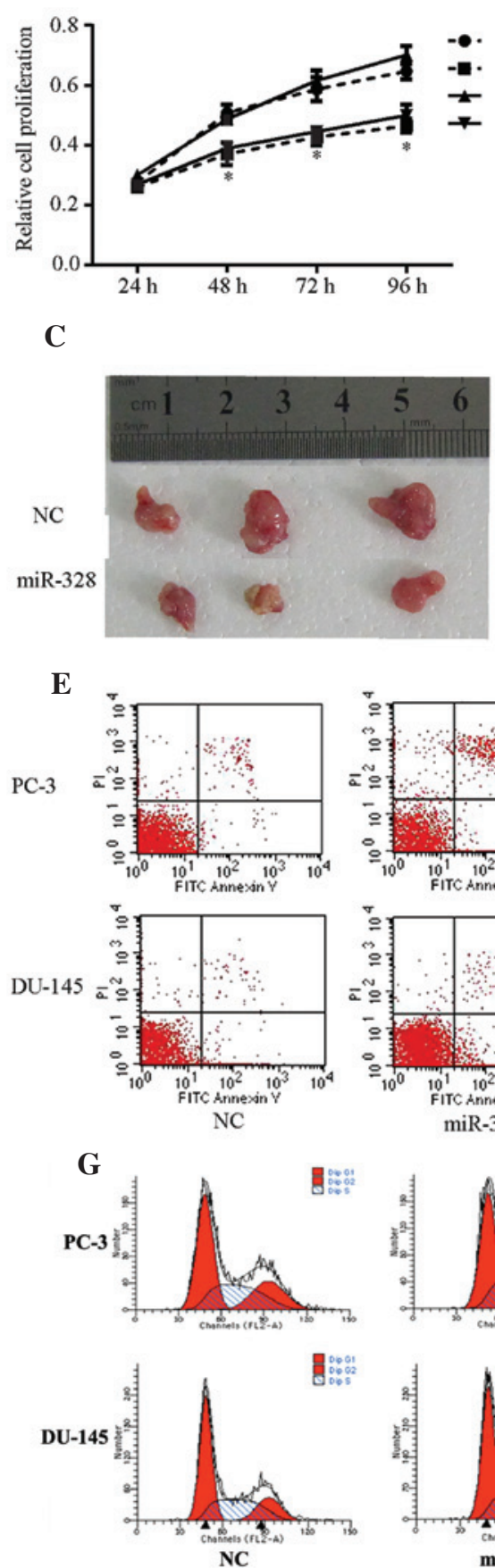

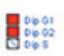

H

Caspase-3

Caspase-9

Bcl-2

GAPDH
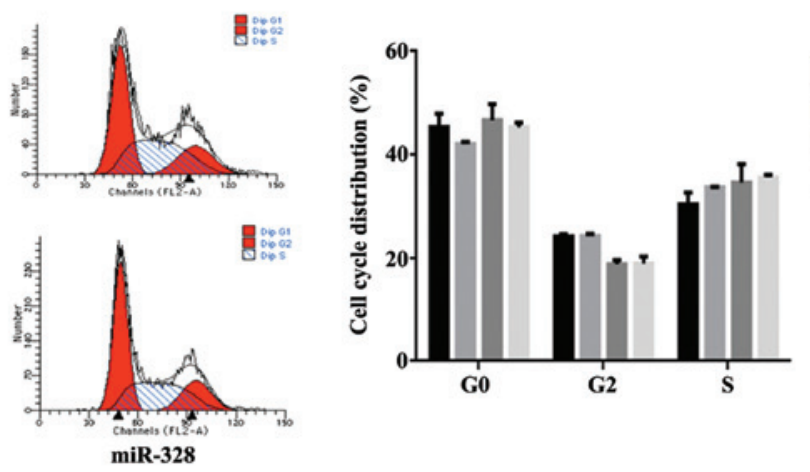

F

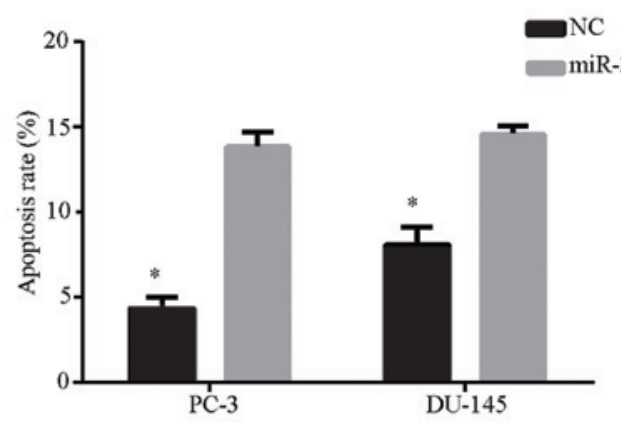

B

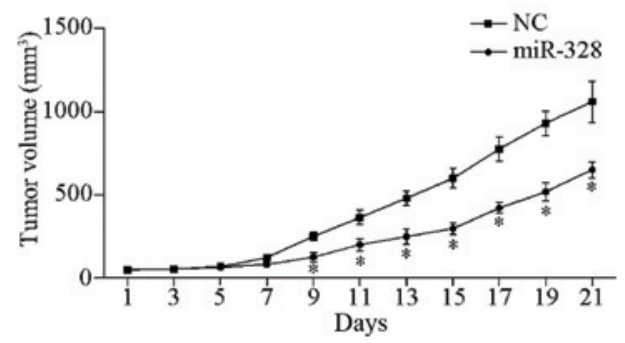

D
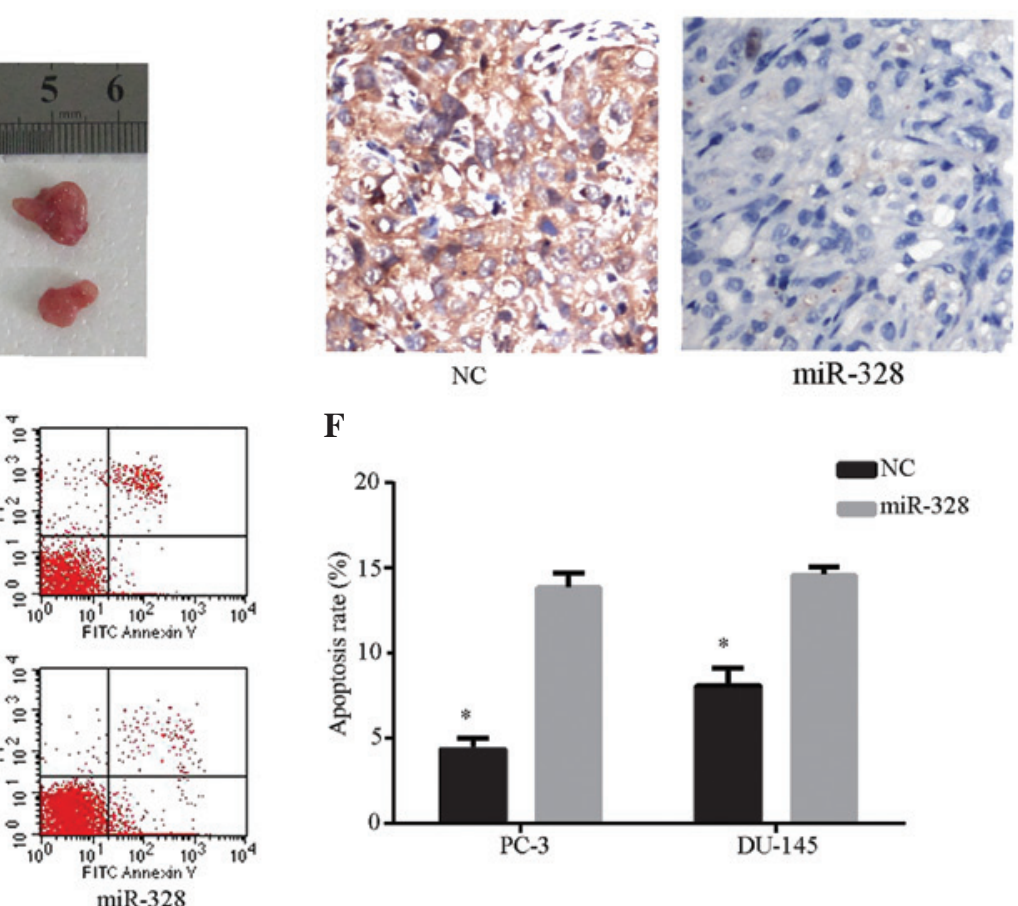

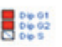

NC miR-328

NC miR-328

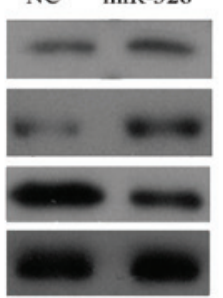

DU-145

Figure 3. miR-328 inhibited cell growth and promoted cell apoptosis. (A) Cell proliferation, determined by Cell Counting Kit-8 assay, indicated that overexpression of miR-328 significantly inhibited PC-3 proliferation. (B and C) Subcutaneous xenograft assay demonstrated that miR-328 significantly inhibited tumor growth in vivo. (D) Immunohistochemistry was performed on xenograft tumors following miR-328 exposure. miR-328 reduced p21-activated protein kinase 6 immunostaining in xenograft tumors (magnification, x200). (E and F) Annexin V-FITC PI-stained cells transfected with miR-328 demonstrated a higher rate of apoptosis, as evaluated by flow cytometry. (G) PI-stained cells transfected with miR-328 exhibited no statistical differences in cell cycle. (H) Cleaved caspase-3 and caspase-9 expression levels were increased and bcl-2 expression was decreased following exposure to miR-328 mimics, as evaluated by western blotting. "P<0.05. NC, negative control; miR, microRNA; FITC, fluorescien isothiocyanate; PI, propidium iodide. 
Table I. p21-activated kinase 6 immunostaining in BPH, Pca and CRPC samples.

\begin{tabular}{lcccc}
\hline Sample & Total & Negative & Weak & Intense \\
\hline BPH & 10 & 4 & 6 & 0 \\
Pca & & & & \\
GS $<7$ & 10 & 1 & 5 & 4 \\
GS 7 & 10 & 0 & 7 & 3 \\
GS $>7$ & 10 & 1 & 5 & 4 \\
CRPC & 9 & 0 & 1 & 8 \\
\hline
\end{tabular}

BPH, benign prostatic hyperplasia; Pca, prostate cancer; GS, Gleason score; CRPC, castration-resistant Pca.

A

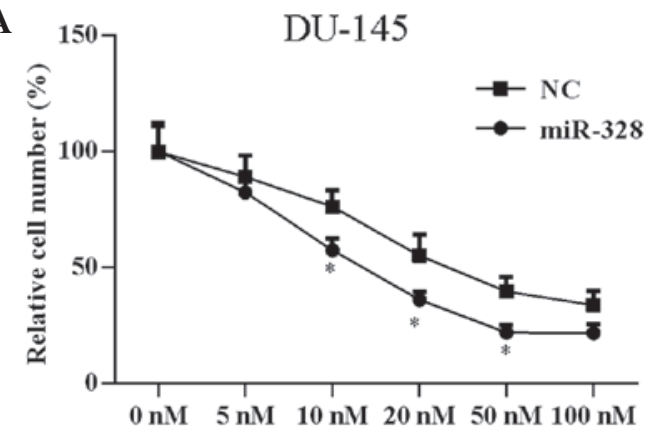

B

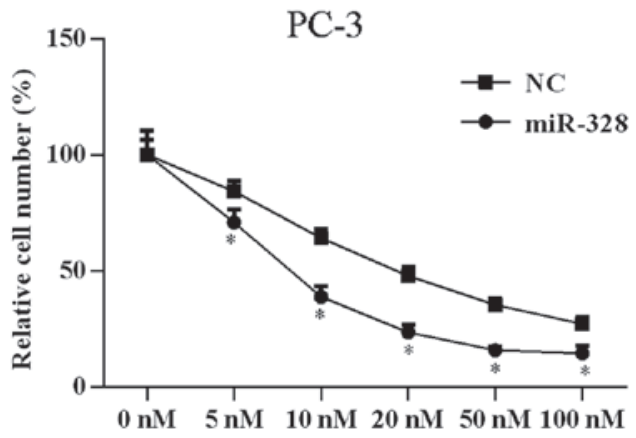

Figure 4. Drug sensitivity test for docetaxel. Following transfection with miR-328 or NC, cells were treated with various concentrations of docetaxel for $24 \mathrm{~h}$ and cell viability was evaluated by Cell Counting kit- 8 analysis. The results demonstrate that miR-328 overexpression enhanced drug sensitivity to docetaxel in (A) DU-145 and (B) PC-3 cells. ${ }^{*} \mathrm{P}<0.05$. miR, microRNA; NC, negative control.

\section{Discussion}

Androgen-deprivation therapy is commonly adopted to treat metastatic Pca; however, the majority of ADPC cases inevitably progress to CRPC (14). Hormone refractory cases and metastasis remain major challenges of Pca, however the mechanisms of these issues remain unclear. Therefore, additional studies are necessary to understand the relevant molecular mechanisms and develop more effective treatment methods. Recent evidence indicates that PAK6 performs critical functions in Pca development $(6,7)$. In the present study, PAK6 overexpression was observed in CRPC tissues, which suggests that PAK6 contributes significantly to the progression of ADPC to CRPC. Furthermore, PAK6 overexpression was observed to be mediated by weak miR-328 expression.

Previous studies have demonstrated that various miRs are involved in Pca development and progression $(15,16)$. miR expression is involved in critical biological processes, including growth, proliferation and apoptosis (17). Pca cell proliferation and apoptosis is closely associated with tumor malignancy and drug resistance, and multiple miRs have been demonstrated to regulate Pca cell proliferation and apoptosis (18). For example, miR-143 arrests cell proliferation by inhibiting extracellular signal-regulated kinase-5 (19), and miR-15 and -205 inhibit proliferation and promote apoptosis in Pca cells by targeting the bcl-2 gene $(20,21)$.

miR-328 is known to be weakly expressed in certain types of cancer, including Pca (22-24). In human breast cancer and glioblastoma cancer stem cells, miR-328 targets breast cancer resistance protein (BCRP/ABCG2) and affects drug disposition $(25,26)$. In bone marrow cells, mir-328 interacts with heterogeneous ribonucleoprotein E2 and affects granulocytic maturation (24). However, miR-328 is also overexpressed in lung adenocarcinoma (27). In non-small cell lung cancer, miR-328 overexpression results in increased cell migration and is associated with brain metastasis (28). In glioma cells, miR-328 targets secreted frizzled-related protein 1 and promotes cell invasion (29). These conflicting roles of miR-328 in different types of cancer indicate the tissue specificity of miR-328 function. In the present study, forced miR-328 overexpression markedly enhanced docetaxel sensitivity, reduced cell proliferation and increased apoptosis in Pca cells without affecting the cell cycle. miR-328 overexpression increases levels of caspase-3 and -9 expression, and decreased bcl-2 expression.

In conclusion, miR-328 is weakly expressed in CRPC, and regulates cell proliferation and apoptosis by targeting PAK6. In addition, upregulated miR-328 expression enhances docetaxel sensitivity, inhibits cell proliferation and promotes cell apoptosis without affecting the cell cycle.

\section{Acknowledgements}

The present study was supported by the National Natural Science Foundation of China (grant nos. 81370849, 81300472 and 81202034), the Natural Science Foundation of Jiangsu Province (grant nos. BL2013032 and BK2012336) and Nanjing City (grant no. 201201053) and Southeast University (grant no. 3290002402), the Science Foundation of Ministry of Education of China (grant no. 20120092120071), the Fundamental Research Funds for the Central Universities and Scientific Research Innovation Project of University in Jiangsu Province (grant no. KYLX_0203).

\section{References}

1. Siegel R, Ma J, Zou Z and Jemal A: Cancer statistics, 2014. CA Cancer J Clin 64: 9-29, 2014.

2. Cookson MS, Roth BJ, Dahm P, Engstrom C, Freedland SJ, Hussain M, Lin DW, Lowrance WT, Murad MH, Oh WK, et al: Castration-resistant prostate cancer: AUA Guideline. J Urol 190: 429-438, 2013.

3. Willard SS and Koochekpour S: Regulators of gene expression as biomarkers for prostate cancer. Am J Cancer Res 2: 650-657, 2012.

4. Yang F,Li X, Sharma M,Zarnegar M,Lim B and Sun Z: Androgen receptor specifically interacts with a novel p21-activated kinase, PAK6. J Biol Chem 276: 15345-15353, 2001. 
5. Lee SR, Ramos SM, Ko A, Masiello D, Swanson KD, Lu ML and Balk SP: AR and ER interaction with a p21-activated kinase (PAK6). Mol Endocrinol 16: 85-99, 2002.

6. Kaur R, Yuan X, Lu ML and Balk SP: Increased PAK6 expression in prostate cancer and identification of PAK6 associated proteins. Prostate 68: 1510-1516, 2008.

7. Schrantz N, da Silva Correia J, Fowler B, Ge Q, Sun Z and Bokoch GM: Mechanism of p21-activated kinase 6-mediated inhibition of androgen receptor signaling. J Biol Chem 279: 1922-1931, 2004.

8. Wen X, Li X, Liao B, Liu Y, Wu J, Yuan X, Ouyang B, Sun Q and Gao X: Knockdown of p21-activated kinase 6 inhibits prostate cancer growth and enhances chemosensitivity to docetaxel. Urology 73: 1407-1411, 2009.

9. Zhang M, Siedow M, Saia G and Chakravarti A: Inhibition of p21-activated kinase 6 (PAK6) increases radiosensitivity of prostate cancer cells. Prostate 70: 807-816, 2010.

10. Ayub SG, Kaul D and Ayub T: Microdissecting the role of microRNAs in the pathogenesis of prostate cancer. Cancer Genet 208: 289-302, 2015.

11. Chua JH, Armugam A and Jeyaseelan K: MicroRNAs: Biogenesis, function and applications. Curr Opin Mol Ther 11: 189-199, 2009.

12. Xu B, Niu X, Zhang X, Tao J, Wu D, Wang Z, Li P, Zhang W, Wu H, Feng N, et al: miR-143 decreases prostate cancer cells proliferation and migration and enhances their sensitivity to docetaxel through suppression of KRAS. Mol Cell Biochem 350: 207-213, 2011

13. Epstein JI, Allsbrook WE Jr, Amin MB, Egevad LL and ISUP Grading Committee: The 2005 International Society of Urological Pathology (ISUP) Consensus Conference on Gleason Grading of Prostatic Carcinoma. Am J Surg Pathol 29: 1228-1242, 2005.

14. Feldman BJ and Feldman D: The development of androgen-independent prostate cancer. Nat Rev Cancer 1: 34-45, 2001.

15. Fang YX and Gao WQ: Roles of microRNAs during prostatic tumorigenesis and tumor progression. Oncogene 33: 135-147, 2014

16. McKee TC and Tricoli JV: Epigenetics of prostate cancer. Methods Mol Biol 1238: 217-234, 2015.

17. Di Leva G, Garofalo M and Croce CM: MicroRNAs in cancer. Annu Rev Pathol 9: 287-314, 2014.

18. Deng JH, Deng Q, Kuo CH, Delaney SW and Ying SY: MiRNA targets of prostate cancer. Methods Mol Biol 936: 357-369, 2013.
19. Clapé C, Fritz V, Henriquet C, Apparailly F, Fernandez PL Iborra F, Avancès C, Villalba M, Culine S and Fajas L: miR-143 interferes with ERK5 signaling, and abrogates prostate cancer progression in mice. PLoS One 4: e7542, 2009.

20. Verdoodt B, Neid M, Vogt M, Kuhn V, Liffers ST, Palisaar RJ, Noldus J, Tannapfel A and Mirmohammadsadegh A: MicroRNA-205, a novel regulator of the anti-apoptotic protein $\mathrm{Bcl} 2$, is downregulated in prostate cancer. Int $\mathrm{J}$ Oncol 43: 307-314, 2013.

21. Cimmino A, Calin GA, Fabbri M, Iorio MV, Ferracin M, Shimizu M, Wojcik SE, Aqeilan RI, Zupo S, Dono M, et al: miR-15 and miR-16 induce apoptosis by targeting BCL2. Proc Natl Acad Sci USA 102: 13944-13949, 2005.

22. Wang W, Peng B, Wang D, Ma X, Jiang D, Zhao J and Yu L: Human tumor microRNA signatures derived from large-scale oligonucleotide microarray datasets. Int J Cancer 129: 1624-1634, 2011.

23. Malzkorn B, Wolter M, Liesenberg F, Grzendowski M, Stühler K, Meyer HE and Reifenberger G: Identification and functional characterization of microRNAs involved in the malignant progression of gliomas. Brain pathol 20: 539-550, 2010.

24. Eiring AM, Harb JG, Neviani P, Garton C, Oaks JJ, Spizzo R, Liu S, Schwind S, Santhanam R, Hickey CJ, et al: miR-328 functions as an RNA decoy to modulate hnRNP E2 regulation of mRNA translation in leukemic blasts. Cell 140: 652-665, 2010

25. Pan YZ, Morris ME and Yu AM: MicroRNA-328 negatively regulates the expression of breast cancer resistance protein (BCRP/ABCG2) in human cancer cells. Mol Pharmacol 75: 1374-1379, 2009

26. Li WQ, Li YM, Tao BB, Lu YC, Hu GH, Liu HM, He J, Xu Y and Yu HY: Downregulation of ABCG2 expression in glioblastoma cancer stem cells with miRNA-328 may decrease their chemoresistance. Med Sci Monit 16: HY27-HY30, 2010.

27. Dacic S, Kelly L, Shuai Y and Nikiforova MN: miRNA expression profiling of lung adenocarcinomas: Correlation with mutational status. Mod Pathol 23: 1577-1582, 2010.

28. Arora S, Ranade AR, Tran NL, Nasser S, Sridhar S, Korn RL, Ross JT, Dhruv H, Foss KM, Sibenaller Z, et al: MicroRNA-328 is associated with (non-small) cell lung cancer (NSCLC) brain metastasis and mediates NSCLC migration. Int J Cancer 129: 2621-2631, 2011.

29. Delic S, Lottmann N, Stelzl A, Liesenberg F, Wolter M, Götze S, Zapatka M, Shiio Y, Sabel MC, Felsberg J, et al: MiR-328 promotes glioma cell invasion via SFRP1-dependent Wnt-signaling activation. Neuro-oncol 16: 179-190, 2014. 